\title{
Functionalized calcium phosphate nanoparticles for biomedical application
}

\author{
Matthias Epple ${ }^{a}$, Anna Kovtun ${ }^{b}$ \\ Inorganic Chemistry and Center for Nanointegration Duisburg-Essen (CeNIDE), University of \\ Duisburg-Essen, Universitaetsstr. 5-7, D-45117 Essen, Germany \\ amatthias.epple@uni-due.de, banna.kovtun@uni-due.de
}

Keywords: calcium phosphate, nanoparticles, gene therapy, drug delivery, transfection.

\begin{abstract}
Calcium phosphate is a natural biomineral and therefore possesses an excellent biocompatibility due to its chemical similarity to human hard tissue (bone and teeth). Calcium phosphate nanoparticles can be precipitated under controlled conditions and used as carrier in biological systems, e.g. to transfer nucleic acids or drugs. Such nanoparticles can also be suitably functionalized with fluorescing dyes, polymeric agents, pro-drugs or activators. The small monodisperse nanoparticles only mildly influence the intracellular calcium level and therefore are not toxic for cells.
\end{abstract}

\section{Introduction}

Nanoparticles represent a key approach to a very wide range of problems. One of their possible applications is the use of nanoparticles as carriers for drugs and nucleic acids in molecular biology and medicine.

In the past years many types of nanocarriers of different chemical nature were developed and tested. Among the most promising examples are inorganic nanoparticles. Although the inorganic nanoparticles cannot compete with viral methods or liposomes in high transfection efficiency, they have often a low toxicity, easy and low-cost preparation, and good storage stability.

Inorganic nanoparticles for biological application may consist of different compounds, e.g. gold [1], silver [2], magnetite [3, 4], silica [5], carbon [6], manganous phosphate [7, 8] and calcium phosphate [9-11]. Among this wide range of materials, calcium phosphates have special properties: as natural biominerals they possess outstanding biocompatibility and biodegradability. Calcium and phosphate are very important inorganic components of biological hard tissues, e.g. bones and teeth [12]. They are also involved in the regulation of many processes, such as Ca-dependent intracellular signaling pathways or maintenance of salt balance of the organism. In the form of geological minerals, calcium phosphates often form large single crystals. In contrast, biological calcium phosphates usually occur as nanocrystals because their formation takes place under mild conditions. They often contain different substitutions such as sodium, magnesium, or carbonate [12-14]. An important parameter for the description of different calcium phosphates is the molar $\mathrm{Ca} / \mathrm{P}$ ratio and solubility. Most types of calcium phosphates are insoluble or only partially soluble in water, but all are soluble in acids.

\section{Nanoparticles for gene therapy}

Calcium phosphate particles were already used by many groups for drug delivery and gene therapy. A scheme of different nanoparticulate systems is shown in Fig. 1. These systems include calcium phosphate as carrier for nucleic acids delivery, i.e. DNA and RNA, and calcium phosphate as a matrix for drug delivery and clinical imaging.

As carriers of nucleic acids, calcium phosphate nanoparticles have been widely used for more than 35 years. The interaction between calcium phosphate and nucleic acid occurs presumably due to the affinity of calcium to the phosphate backbone in nucleic acids [15], thus making the nucleotide sequence of the nucleic acid unimportant, but taking into account its length. 
The first precipitation method for transfection with calcium phosphates was described by Graham and van der Eb in 1973 [10]. The precipitation occurs in situ and includes a subsequent mixing of calcium chloride solution, DNA, and phosphate-buffered saline solution [9, 11]. This results in the formation of polydisperse nano- and microparticles of calcium phosphate and DNA [16] (Figure 1). This a method has distinct drawbacks such as the polydispersity of the particles, little control of the experimentalist over their size and morphology (and, therefore a poor reproducibility of the method), and a short storage time for the precipitates (they have to be used immediately for transfection). However, with optimized parameters a rather high efficiency can be achieved. Jordan and co-workers found that the main parameters that must be taken into account are the concentrations of calcium, phosphate, and DNA. Spontaneous precipitation of nanoparticles occurs only if concentrations of calcium and phosphate are high enough to ensure supersaturation. On the other hand, very high concentrations of DNA $\left(>50 \mu \mathrm{g} \mathrm{mL}^{-1}\right)$ blocked the formation of precipitates, whereas minor concentration resulted in a rapid particle formation within $1 \mathrm{~min}$ [11].

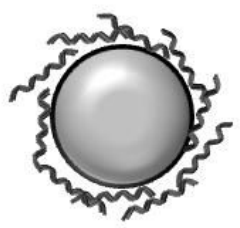

Welzel et al.

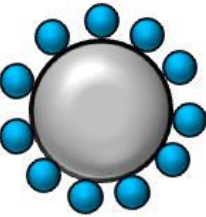

Cheng et al. nanoparticles with cisplatin

Calcium phosphate nanoparticles for gene delivery

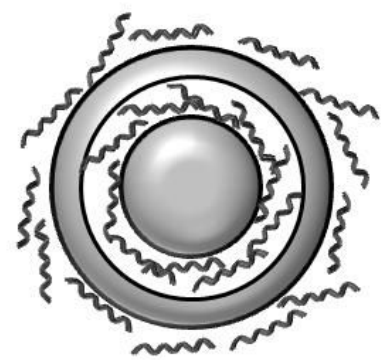

Sokolova et al.

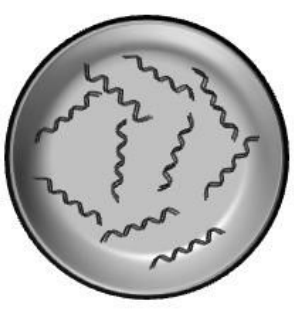

Bisht et al.

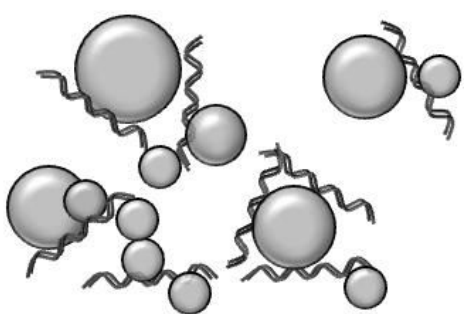

Graham et al.

\section{Calcium phosphate nanoparticles for drug delivery}

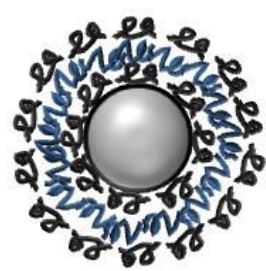

Schwiertz et al. nanocapsules

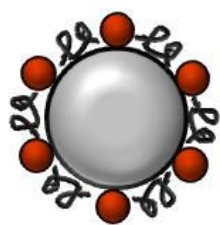

Schwiertz et al. Ganesan et al. nanoparticles with fluorescent dye

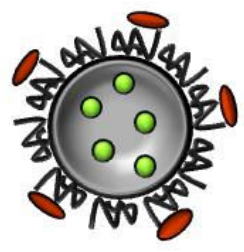

Morgan et al. nanoparticles with incorporated fluoresceine, PEG and ceramide

Figure 1: Schematic representation of different calcium phosphate-based carrier systems.

Welzel et al. developed a method of controlled precipitation of spherical calcium phosphate nanoparticles functionalized with DNA in an aqueous solution [17]. The formation of the crystals was inhibited by functionalization with DNA. Such nanoparticles had a size up to $200 \mathrm{~nm}$ with a negative zeta potential up to $-30 \mathrm{mV}[17,18]$. However, transfection experiments with such particles showed only a low efficiency. 
By improvement of this method Sokolova et al. developed multi-shell particles where the inner DNA shell was protected against nuclease attack in the extra- and intracellular compartments [19]. Such nanoparticles possess additional layers of calcium phosphate and DNA on the surface of previously precipitated particle. The outer calcium phosphate shell provides protection for DNA, and the outer DNA shell provides electrostatic and steric stabilization of the particles.

Bisht et al. prepared calcium phosphate nanoparticles from microemulsions with a size range of 30$40 \mathrm{~nm}$ [20]. In these particles, DNA was encapsulated into the calcium phosphate core and thus protected from the degradation by nucleases. However, these particles were not stable and strongly aggregated within $24 \mathrm{~h}$ to $80-90 \mathrm{~nm}$.

Elements such as magnesium and aluminum are known to inhibit the growth of calcium phosphate crystals. Magnesium has chemical properties very similar to those of calcium and can also partially replace this ion in many crystals [21]. Chowdhury et al. successfully incorporated fibronectin and $\mathrm{Mg}^{2+}$ into calcium phosphate particles in order to achieve a higher transfection efficiency than the classical precipitation method [7]. They reached circa ten times higher efficiency for the plasmid expression then that shown by classic calcium phosphate transfection method. Bhakta et al. used magnesium phosphate nanoparticles to transfect HeLa cells and achieved a transfection efficiency comparable to that of Polyfect ${ }^{\circledR}[22]$.

The nanoparticles can also be functionalized with siRNA or oligonucleotides as well as DNA [23]. In this case the successful transfection will lead to the inhibition of the production of the desired protein due to its mRNA cleavage.

\section{Intracellular transport of the particles for gene delivery}

Although the classic calcium phosphate transfection method has been widely used since many years, the exact mechanism of the transfection, i.e. of the DNA entry, intracellular release and subsequent expression, still remains still unclear.

To reach a successful transfection, DNA should enter the cell by endocytosis and then enter the nucleus (Fig. 2). Thus, the best transfection efficiency is usually achieved by particles with a size around $40-70 \mathrm{~nm}$. Loyter et al. observed by transmission electron microscopy that calcium phosphate particles enter the cell by endocytosis after about 1-2 $\mathrm{h}$ of the transfection [24]. These data were also confirmed for the TRITC-BSA-marked (tetramethylrhodamine isothiocyanatebovine serum albumin) nanoparticles by Sokolova and co-workers [25]. The particles should escape the endosome before its cytoplasmatic fusion with lysosome to prevent the degradation of DNA. In the lysosome, the proton pump induces a $\mathrm{pH}$ drop followed by the dissolution of calcium phosphate and the release of DNA. This DNA will later be cleaved by lysosomal nucleases. Therefore, DNA needs to leave the lysosome before the protective calcium phosphate will be completely dissolved and it becomes available for nucleases. Nevertheless, Maitra indicated that partial dissolution of calcium phosphate can destabilize the lysosomal membrane and enhance the release of the remaining nanoparticle and DNA into the cytoplasm [26]. There DNA will move into the direction of the nucleus. However, the mechanism of nuclear targeting of DNA and its penetration to the nucleus is still unclear. Sokolova et al. showed that after $6 \mathrm{~h}$ of transfection the nanoparticles were concentrated in the nuclear region [25]. We do not know whether the complete nanoparticle itself penetrates the nuclear membrane, or whether the DNA enters the nucleus alone. Orrantia and Chang indicated that nanoparticles can also enter the nucleus [27]. However, the nanoparticles are usually too large to penetrate the nuclear membrane, thus their nuclear entry is still questionable. In the nucleus DNA can be directly expressed, resulting in a transient transfection and synthesis of the desired protein (that will disappear after several cell division cycles), or intercalate into the genome resulting in a stable transfection. Fig. 2 shows a schematic representation of the transfection mechanism. 


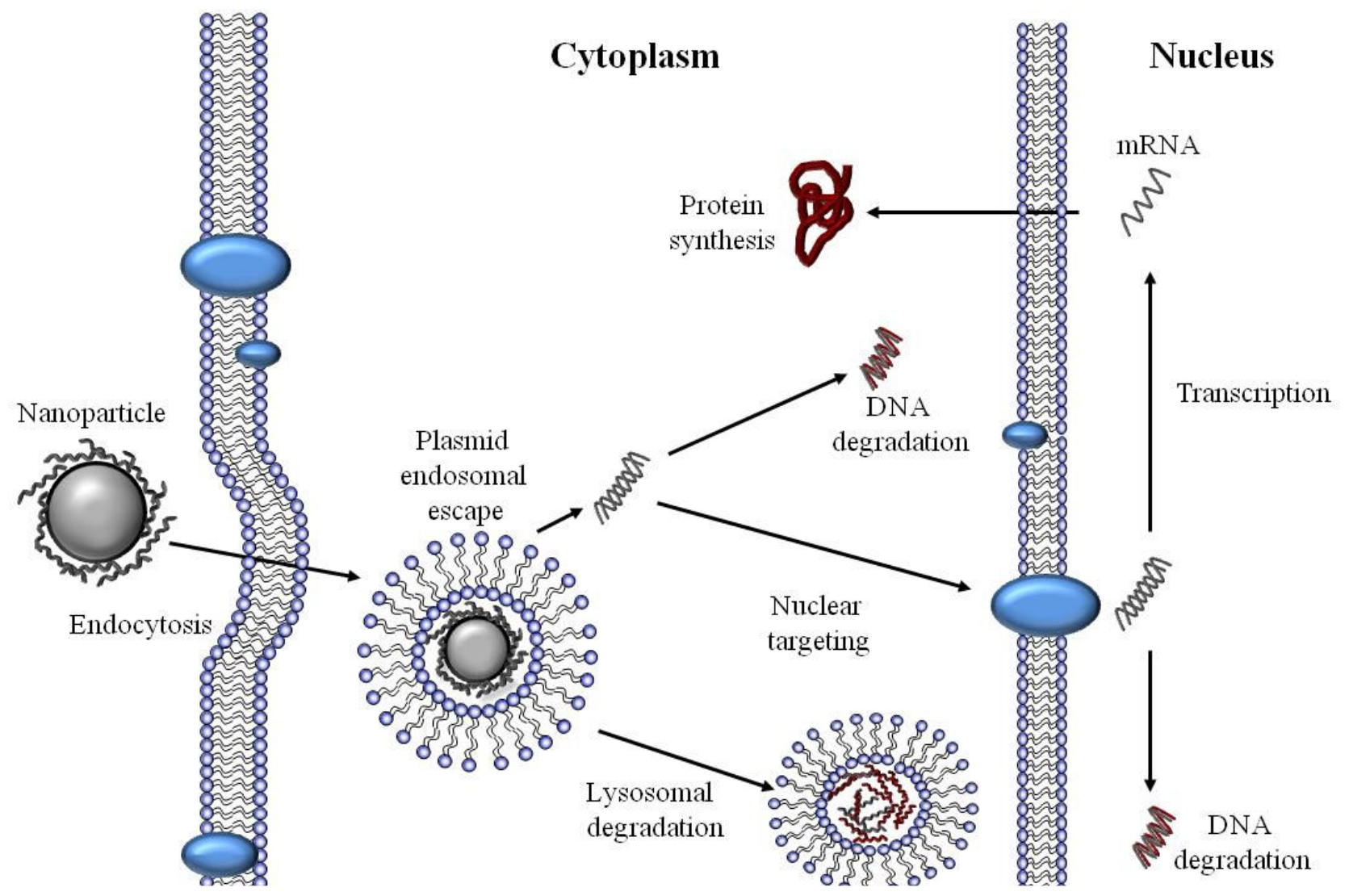

Figure 2: Schematic representation of transfection mechanism.

The influence of calcium exerted on the cells after the calcium phosphate-based transfection is also an important question. Usually the intracellular calcium concentration is maintained on a very low level (lower than $10^{-10} \mathrm{M}$ ) [21] and its increase could lead to the disturbance of calcium-dependent signaling pathways or even intracellular osmotic balance, which can be fatal for the cell. Ewence et al. reported that calcium phosphate crystals from the atherosclerotic plaques induced a rapid increase of the intracellular calcium concentration which caused the death of $50 \%$ of the investigated vascular smooth muscle cells [28]. They found that this increase occurred due to the dissolution of calcium phosphate crystals in the lysosomes which caused the rupture of the latter, an overall increase of calcium concentration in the cytoplasm, and, finally, cell death. The same results were obtained by Neumann et al. comparing different transfection methods [29]. The monitoring of intracellular calcium concentration using the calcium-sensitive dye Fura-2 showed that DNAfunctionalized single- and multi-shell nanoparticles led to a minor and local increase of the intracellular calcium concentration (presumably in lysosomes, where nanoparticles are dissolved). Simultaneously, the application of the standard phosphate precipitation method led to high calcium spikes in the whole cytoplasm of cells. This indicated that particles were dissolved in lysosomes, lead to their rupture and the release of excessive calcium into the cytoplasm. This calcium should be pumped into intracellular calcium stores (endoplasmatic reticulum and mitochondria) or out of the cell. However, above a critical calcium concentration, it can become impossible for the cell to pump out excessive calcium which causes cell death. Thus, after the standard calcium phosphate transfection method, only 50-65\% of cells survived whereas after the application of the DNAfunctionalized calcium phosphate nanoparticles, the viability of cells did not significantly change. 


\section{Nanoparticles for drug delivery}

Drug delivery is another promising application of nanocarriers. They can serve as a matrix for a more complex system for the therapeutic application. In this case the nanoparticles can be used to simultaneously achieve different goals: Drug delivery, imaging and therapeutic activation of the carrier itself [30]. The composition of the nanoparticles varies depending on the target tissue, but in general, four main components may be distinguished [31]:

1. An inorganic or organic core, which usually acts only as a carrier (lipid or polymeric nanoparticles), but can also have therapeutic properties itself (e.g. magnetic nanoparticles for hyperthermia therapy destroy the tumor cells [32],

2. one or several drugs or pro-drugs as active component of nanoparticle. These can be peptides, proteins, oligonucleotides, etc.,

3. the targeting compound which enables specific delivery of the particle to the cell or tissue of interest (e.g. antibodies),

4. a stabilizing component which can furthermore facilitate its penetration through the vessel epithelium or cell membrane.

Furthermore, particles may sometimes contain even more additional components such as imaging agents or tumor-specific cleavable linkages for further activation. For example, the nanoparticle core can be visualized by the integration of lanthanides [33], fluorescing dyes such as Cy3 [34] or 5,10,15,20-tetrakis(4-phosphonooxyphenyl)porphine ( $p$ TPPP) [35]. Calcium phosphate may also be used as a template for a more complex structure. Schwiertz et al. used calcium phosphate as core for the synthesis of polymeric nanocapsules by the layer-by-layer technique [36]. These nanocapsules could then loaded with a drug and used for drug delivery in vivo.

The other important parameters for particles are the circulation half-life time, the stability in the blood stream, and the surface charge for the penetration of the blood vessel epithelium and cellular membrane.

The properties of the particles strongly depend on their application and target tissue. One of the most pronounced examples is the particle size. In the case of calcium phosphate nanoparticles it can be easily controlled by stabilizing agents such as polymers or nucleic acids. Particles smaller than $100 \mathrm{~nm}$ are hardly recognized by the immune system, can be easily taken up by cells and are big enough to escape the renal filtration, thus providing longer circulating half-life and thus better passive targeting of tumor tissues [37]. On the other hand, when the targeted cells are mononuclear phagocytes, the micrometer size of the particles is preferable. For example Krishnamachari and Salem performed the activation of dendritic cells by oligonucleotides [38]. The oligonucleotide uptake led to the maturation of dendritic cells and to a better presentation of an otherwise undetectable antigen, following by an effective immune response.

Calcium phosphate is the mineral of bone, therefore it shows excellent biocompatibility and can be easily eliminated by low $\mathrm{pH}$, as in case of lysosomes or environment of solid tumors. The functionalization of the nanoparticles with different bioactive molecules enables in vivo therapy, i.e. Cheng et al. synthesized cisplatin-conjugated nanoparticles for chemotherapy [39] and Ramachadran et al. performed the synthesis of insulin-functionalized particles for oral insulin delivery [39, 40]. Kester et al. produced stable calcium phosphate nanoparticles functionalized with the anticancer drug ceramide [41]. The further functionalization with PEG resulted in stable particles with a positive surface charge which should be easily taken up by cells [41]. 
Schwiertz et al. prepared particles functionalized with polymer and 5,10,15,20-tetrakis(3hydroxyphenyl)porphyrin (mTHPP), a compound which is applied as photosensitizer in photodynamic therapy (PDT) [42].

In conclusion, the calcium phosphate nanoparticles represent an easily modified solid carrier for more complex delivery system, therefore they represent a very promising approach for the delivery of genes or drugs of different chemical nature.

\section{References}

[1] M. Tsoli, H. Kuhn, W. Brandau, H. Esche, G. Schmid: Small Vol. 1 (2005), p. 841.

[2] J. Zhang, J. Malicka, I. Gryczynski, J. R. Lakowicz: Anal. Biochem. Vol. 330 (2004), p. 81.

[3] A. del Campo, T. Sen, J. P. Lellouche, I. J. Bruce: Journal of Magnetism and Magnetic Materials Vol. 293 (2005), p. 33.

[4] Y. Fukumori, H. Ichikawa: Adv. Powder Techn. Vol. 17 (2006), p. 1.

[5] M. Boissière, J. Allouche, C. Chanéac, R. Brayner, J. M. Devoisselle, J. Livage, T. Coradin: Int. J. Pharm. Vol. 344 (2007), p. 128.

[6] Z. Liu, M. Winters, M. Holodniy, H. Dai: Angew. Chem. Vol. 119 (2007), p. 2069.

[7] E. H. Chowdhury, M. Kunou, M. Nagaoka, A. K. Kundu, T. Hoshiba, T. Akaike: Gene Vol. 341 (2004), p. 77.

[8] E. H. Chowdhury, F. T. Zohra, S. Tada, C. Kitamura, T. Akaike: Anal. Biochem. Vol. 335 (2004), p. 162.

[9] P. Girard, L. Porte, T. Berta, M. Jordan, F. Wurm: Cytotechnology Vol. 35 (2001), p. 175.

[10] F. L. Graham, A. J. van der Eb: Virology Vol. 52 (1973), p. 456.

[11] M. Jordan, A. Schallhorn, F. M. Wurm: Nucleic Acids Res. Vol. 24 (1996), p. 596.

[12] S. V. Dorozhkin, M. Epple: Angew. Chem. Int. Ed. Vol. 41 (2002), p. 3130.

[13] C. Rey, C. Combes, C. Drouet, H. Sfihi, A. Barroug: Mater. Sci. Eng. C Vol. 27 (2007), p. 198.

[14] C. Rey, C. Combes, C. Drouet, A. Lebugle, H. Sfihi, A. Barroug: Mat.-wiss. u. Werkstofftech. Vol. 38 (2007), p. 996.

[15] M. Okazaki, Y. Yoshida, S. Yamaguchi, M. Kaneno, J. C. Elliott: Biomaterials Vol. 22 (2001), p. 2459.

[16] Y. W. Yang, J. C. Yang: Biomaterials Vol. 18 (1997), p. 213.

[17] T. Welzel, I. Radtke, W. Meyer-Zaika, R. Heumann, M. Epple: J. Mater. Chem. Vol. 14 (2004), p. 2213.

[18] V. Sokolova, O. Prymak, W. Meyer-Zaika, H. Cölfen, H. Rehage, A. Shukla, M. Epple: Mat.-wiss. u. Werkstofftech. Vol. 37 (2006), p. 441.

[19] V. V. Sokolova, I. Radtke, R. Heumann, M. Epple: Biomaterials Vol. 27 (2006), p. 3147.

[20] S. Bisht, G. Bhakta, S. Mitra, A. Maitra: Int. J. Pharm. Vol. 288 (2005), p. 157.

[21] M. C. F. Magalhaes, P. A. A. P. Marques, R. N. Correia, in: Biomineralization: Medical aspects of solubility, edited by E. Königsberger, L. C. Königsberger / John Wiley \& Sons (2006).

[22] G. Bhakta, S. Mitra, A. Maitra: Biomaterials Vol. 26 (2005), p. 2157.

[23] V. Sokolova, A. Kovtun, O. Prymak, W. Meyer-Zaika, E. A. Kubareva, E. A. Romanova, T. S. Oretskaya, R. Heumann, M. Epple: J. Mater. Chem. Vol. 17 (2007), p. 721.

[24] A. Loyter, G. Scangos, D. Juricek, D. Keene, F. H. Ruddle: Exp. Cell Res. Vol. 139 (1982), p. 223.

[25] V. Sokolova, A. Kovtun, R. Heumann, M. Epple: J. Biol. Inorg. Chem. Vol. 12 (2007), p. 174.

[26] A. Maitra: Expert Rev. Mol. Diagn. Vol. 5 (2005), p. 893.

[27] E. Orrantia, P. L. Chang: Exp. Cell. Res. Vol. 190 (1990), p. 170.

[28] A. E. Ewence, M. Bootman, H. L. Roderick, J. N. Skepper, G. McCarthy, M. Epple, M. Neumann, C. M. Shanahan, D. Proudfoot: Circ. Res. Vol. 103 (2008), p. e28. 
[29] S. Neumann, A. Kovtun, I. D. Dietzel, M. Epple, R. Heumann: Biomaterials Vol. 30 (2009), p. 6794.

[30] M. Liong, J. Lu, M. Kovochich, T. Xia, S. G. Ruehm, A. E. Nel, F. Tamanoi, J. I. Zink: ASC Nano Vol. 2 (2008), p. 889.

[31] P. R. Gil, W. J. Parak: ASC Nano Vol. 2 (2008), p. 2200.

[32] V. I. Shubayev, T. R. Pisanic, S. Jin: Adv. Drug Deliv. Rev. Vol. 61 (2009), p. 467.

[33] S. Padilla Mondejar, A. Kovtun, M. Epple: J. Mater. Chem. Vol. 17 (2007), p. 4153.

[34] H. S. Muddana, T. T. Morgan, J. H. Adair, P. J. Butler: Nano Lett. (2009), p. 1559.

[35] K. Ganesan, M. Epple: New J. Chem. Vol. 32 (2008), p. 1326.

[36] J. Schwiertz, W. Meyer-Zaika, L. Ruiz-Gonzalez, J. M. Gonzales-Calbet, M. Vallet-Regi, M. Epple: J. Mater. Chem. Vol. 18 (2008), p. 3831.

[37] F. Yuan: Semin. Radiat. Oncol. Vol. 8 (1998), p. 164.

[38] Y. Krishnamachari, A. K. Salem: Adv. Drug Deliv. Rev. Vol. 61 (2009), p. 205.

[39] X. Cheng, L. Kuhn: Int. J. Nanomed. Vol. 2 (2007), p. 667.

[40] R. Ramachandran, W. Paul, C. P. Sharma: J. Biomed. Mater. Res. Part B: Appl. Biomater. Vol. 88B (2009), p. 41.

[41] M. Kester, Y. Heakal, T. Fox, A. Sharma, G. P. Robertson, T. T. Morgan, E. I. Altinoglu, A. Tabakovic, M. R. Parette, S. M. Rouse, V. Ruiz-Velasco, J. H. Adair: Nano Lett. Vol. 8 (2008), p. 4116.

[42] J. Schwiertz, A. Wiehe, S. Gräfe, B. Gitter, M. Epple: Biomaterials Vol. 30 (2009), p. 3324. 